\title{
Amino-sugar Transport Systems of Escherichia coli K12
}

\author{
By M. C. JONES-MORTIMER AND H. L. KORNBERG* \\ Department of Biochemistry, University of Cambridge, Tennis Court Road, \\ Cambridge CB2 $1 Q W$
}

(Received 20 September 1979)

\begin{abstract}
Glucosamine, mannose and 2-deoxyglucose enter Escherichia coli by the phosphotransferase system coded for by the gene $p t s M$. The glucosamine- and mannose-negative, deoxyglucoseresistant phenotype of $p t s M$ mutants can be suppressed by a mutation mapping near $p t s G$ that allows constitutive expression of the glucose phosphotransferase coded for by the gene $p t s G$.

$N$-Acetylglucosamine enters $E$. coli by two distinct phosphotransferase systems (White, 1970). One of these is the PtsM system, the other is coded for by a gene which maps near the $n a g A, B$ genes at about min 15 on the $E$. coli chromosome. We propose that this gene be designated pts $N$. Strains with either of these components of the phosphotransferase system will utilize $N$-acetylglucosamine as sole carbon source.
\end{abstract}

\section{INTRODUCTION}

Many, though not all, sugars enter Escherichia coli by a phosphotransferase mechanism (Kundig et al., 1964). For transport of a sugar to occur in this manner the phosphate group of phosphoenolpyruvate is transferred, in a reaction catalysed by Enzyme I, to a small histidine-containing protein, HPr. The phosphate group is then transferred from the phosphorylated HPr to the appropriate sugar, which appears inside the cell as the sugar phosphate. The latter, sugar-specific, transfer reaction is catalysed by one of a series of enzymes generically known as Enzymes II. However, the specificity for their sugar substrates exhibited by Enzymes II is not absolute (for review, see Kornberg, 1976).

The gene $p t s M$ [originally called $m p t$ by Curtis \& Epstein (1975) and $p t s X$ by Kornberg \& Jones-Mortimer (1975)] codes for such an Enzyme II. This Enzyme II (PtsM system) effects the transport of a number of hexoses with the D-arabino configuration. Thus, Curtis \& Epstein (1975) have shown that glucose, mannose, glucosamine, 2-deoxyglucose and $N$-acetylmannosamine are all substrates for this system and we (Kornberg \& JonesMortimer, 1975) have shown that fructose is too. In the present paper, we show that one of the two phosphotransferases for $N$-acetylglucosamine described by White (1970) is that specified by $p t s M$ and that the other phosphotransferase for $N$-acetylglucosamine maps (as White predicted) near the genes for $N$-acetylglucosamine-6-phosphate deacetylase (nag $A$ ) and glucosamine-6-phosphate deaminase ( $\operatorname{nag} B$ ), at about min 15 on the $E$. coli chromosome. We propose that the gene for this phosphotransferase be described by the symbol pts $N$.

We suggested (Kornberg \& Jones-Mortimer, 1975) that glucosamine might also enter E. coli by the PtsG system (formerly described as Umg or Gpt), the major phosphotransferase for glucose, provided that this system was expressed constitutively or had previously been induced. We have confirmed this suggestion by selecting PtsG-constitutive mutants as spontaneous glucosamine-positive derivatives of a pts $M$ strain, and by showing that glucose-negative derivatives of such a strain $(p t s G)$ become simultaneously glucosaminenegative (and mannose-negative) and deoxyglucose-resistant. 


\section{Table 1. Strains of Escherichia coli used}

All strains are Hfr PO2A, except K1.1.4 which is PO1, and 5K-C600 and HK360 which are F .

\begin{tabular}{|c|c|c|}
\hline Strain & Genotype & Reference or source \\
\hline HK 360 & purB ptsG ptsM ptsF umgC & Laboratory stock \\
\hline DF $1071.2 B$ & eda & Faik et al. (1971) \\
\hline K1.1.4 & thy $A$ met $B$ pps glt $A$ & Laboratory stock \\
\hline $5 \mathrm{~K}-\mathrm{C} 600$ & thr leu lac hsdR supE rps & S. Glover (Jones-Mortimer, 1973) \\
\hline JM 1077 & thy $A$ hisgnd $\triangle$ fda ${ }^{\mathrm{ts}}$ pts $M$ mglP galK ptsF & Henderson et al. (1977) \\
\hline JM 1080 & thy A hisgnd $\Delta$ fda ${ }^{\text {ts }}$ pts $M$ mglP galK ptsF galP & JM 1077 (Henderson et al., 1977) \\
\hline $\mathrm{JM} 1110$ & thy $A$ hisgnd $\Delta$ fda ${ }^{\text {ts }}$ pts $M$ mglP galK ptsF galP umg $C$ & JM1080 (Glucosamine ) \\
\hline JM1118 & thy $A$ hisgnd $\mathrm{fda}{ }^{\text {ts }}$ pts $M$ mglP galK ptsF galP umgC ptsG & JM1110 (Glucose" on glycerol) \\
\hline JM1120 & thy $A$ hisgnd ${ }^{\Delta} f d a^{\text {t. }}$ pts $M$ mglP galK ptsF pts $N$ & $\begin{array}{l}\text { JM1077 ( N-Acetylglucosamine } \mathrm{R}^{\mathrm{R}} \\
\text { on glycerol) }\end{array}$ \\
\hline JM1121 & thy $A$ hisgnd ${ }^{\Delta} f^{\text {ts }}$ mglP galK ptsF pts $N$ eda & $\begin{array}{l}\text { PI .DF 1071.2B } \times \text { JM1120: } \\
\text { Glucosamine }\end{array}$ \\
\hline JM1123 & thy $A$ hisgnd ${ }^{\Delta} f_{d a}{ }^{t s} p t s M$ mglP ptsF pts $N$ glt $A$ & P1.K1.1.4×JM1120: Galactose \\
\hline JM1138 & thy A hisgnd ${ }^{\Delta} f_{d a}^{\text {ts }}$ mglP galK ptsF galP umgC ptsG eda & $\begin{array}{l}\text { P1 . DF 1071.2B } \times \text { JM1118; } \\
\text { Glucosamine }^{+}\end{array}$ \\
\hline JM1141 & $\begin{array}{l}\text { thy } A \text { hisgnd } \Delta \text { fda }^{\text {ts }} \text { pts } M \text { mglP galK ptsF galP umgC } \\
\text { ptsG eda }\end{array}$ & $\begin{array}{l}\text { JM1138 (Glucosamine }{ }^{k} \text { on } \\
\text { glycerol) }\end{array}$ \\
\hline JM1156 & thy A hisgnd ${ }^{\perp} f d a^{\text {ts }}$ pts $M$ mglP galK ptsF galP umgC ptsN & $\begin{array}{l}\text { JM1110 ( N-Acetylglucosamine } \\
\text { on glycerol) }\end{array}$ \\
\hline JM1111 & cysI hisgnd ${ }^{\prime}$ fda ${ }^{\text {ts }}$ ptsM mglP galK uhpC srlA & from JM1077 in four stages \\
\hline JM 1158 & cysI hisgnd ${ }^{\Delta} f_{d a}{ }^{t g}$ ptsM mglP galK uhpC srlA pts $N$ & $\begin{array}{l}\text { JM1111 ( N-Acetylglucosamine } \\
\text { on glycerol) }\end{array}$ \\
\hline JM1161 & cysI hisgnd ${ }^{د}$ fda $^{\text {ts }}$ ptsM mglP galK uhpC srlA supE & $\begin{array}{l}\text { P1.5K-C } 600 \times \mathrm{JM} 1158 \\
N \text {-Acetylglucosamine }\end{array}$ \\
\hline JM1165 & cysI hisgnd ${ }^{\Delta}$ fda $^{t_{\mathrm{s}}}$ ptsM mglP galK uhpC srlA supE ptsN! & JM1161 ( $N$-Acetylglucosamine ${ }^{\mathrm{R}}$ \\
\hline JM1166 & cysI hisgnd ${ }^{\mathrm{f}} \mathrm{fda}^{\mathrm{ts}}$ ptsM mglP galK uhpC srlA supE ptsNj & on glycerol) \\
\hline JM1179 & thr leu lac hsdR supE ptsN rps & $\begin{array}{l}5 \mathrm{~K}-\mathrm{C} 600(2-\mathrm{Deoxy}-2 \text {-iodoacet- } \\
\text { amidoglucose } \mathrm{R}^{\mathrm{R}} \text { on glucose) }\end{array}$ \\
\hline
\end{tabular}

\section{METHODS}

Cultures were grown on the minimal medium of Ashworth \& Kornberg (1966) or on Oxoid nutrient broth (some batches of which proved to inhibit the strains). Growth rates were determined using an EEL nephelometer. The uptake of ${ }^{14} \mathrm{C}$-labelled sugars (from a concentration of $50 \mu \mathrm{M}$ ) was measured by a modification of the method of Kornberg (1972) in which the sugar was added to cultures growing exponentially with lactate as the carbon source at $20^{\circ} \mathrm{C}$. Pl-mediated transduction was carried out by the method of Lennox (1955). The strains of $E$. coli employed are listed in Table 1. The genetic symbols are those of Bachmann et al. (1976), except that the additional symbols $p t s N$ and $u m g C$ are used to indicate genes for the $N$-acetylglucosamine phosphotransferase and for the constitutive expression of the PtsG glucose phosphotransferase, respectively. The bacteriophage T4 mutant N82 was a gift from Dr E. McFall, and 2-deoxy-2-iodoacetamidoD-glucose from Dr P. W. Kent.

\section{RESULTS AND DISCUSSION}

Entry of glucosamine into mutants constitutive for the PtsG system

About $10^{8}$ cells of strain JM1080 were plated on solid medium with glucosamine as sole carbon source and incubated at $30^{\circ} \mathrm{C}$ for $3 \mathrm{~d}$. Since this strain is $p t s M$ it does not grow on glucosamine unless the PtsG system is induced. Glucosamine is, however, not an inducer of that system: glucosamine-positive colonies are therefore likely to form PtsG constitutively. Single-colony isolates were made from colonies that appeared on this medium and tested for their ability to grow on solid medium with glucosamine or mannose as carbon source and to take up methyl $\alpha$-D- $\left[{ }^{14} \mathrm{C}\right]$ glucoside from nutrient agar plates also containing that glucose analogue at $10 \mu \mathrm{M}$. One strain, JM1110, which had the desired properties was chosen for subsequent experiments. That strain JM1110, which was shown to be umgC by direct 
Table 2. Mean generation times of E. coli strains on different carbon sources Carbon sources were present at $10 \mathrm{~mm}$, except lactate which was at $20 \mathrm{~mm}$. Mean generation times are expressed in $h$.

\begin{tabular}{|c|c|c|c|c|c|c|c|}
\hline \multirow[b]{2}{*}{ Strain } & \multirow[b]{2}{*}{ Genotype } & \multicolumn{6}{|c|}{ Carbon source } \\
\hline & & Mannitol & Glucose & $\begin{array}{l}\text { Glucos- } \\
\text { amine }\end{array}$ & Mannose & Lactate & $\begin{array}{c}\text { Lactate+ } \\
\text { 2-deoxy- } \\
\text { glucose }\end{array}$ \\
\hline JM1080 & $p t s M p t s G^{+} u m g C^{+}$ & $2 \cdot 0$ & $2 \cdot 0$ & $>48$ & 7 & $2 \cdot 3$ & $2 \cdot 3$ \\
\hline JM1110 & ptsM pts $G^{+} u m g C$ & $2 \cdot 2$ & $2 \cdot 2$ & $2 \cdot 2$ & $2 \cdot 2$ & $2 \cdot 9$ & $8 \cdot 2$ \\
\hline JM1118 & ptsM ptsG umgC & 1.9 & $>24$ & $>24$ & $>24$ & $3 \cdot 0$ & $3 \cdot 0$ \\
\hline JM1138 & $p t s M^{+} p t s G$ umgC & $2 \cdot 5$ & $>48$ & 3.5 & $3 \cdot 0$ & $2 \cdot 6$ & $7 \cdot 0$ \\
\hline
\end{tabular}

Table 3. Uptake of methyl $\alpha-\mathrm{D}-\left[{ }^{14} \mathrm{C}\right]$ glucoside and 2 -deoxy $\left[{ }^{14} \mathrm{C}\right]$ glucose by E. coli strains

\begin{tabular}{|c|c|c|c|c|}
\hline \multirow[b]{2}{*}{ Strain } & \multirow[b]{2}{*}{ Genotype } & \multirow[b]{2}{*}{$\begin{array}{l}\text { Carbon } \\
\text { source }\end{array}$} & \multicolumn{2}{|c|}{$\begin{array}{c}\text { Uptake } \\
{\left[\mathrm{nmol}(\mathrm{mg} \mathrm{dry} \mathrm{wt})^{-1} \mathrm{~min}^{-1}\right]}\end{array}$} \\
\hline & & & $\begin{array}{c}\text { Methyl } \\
\alpha \text {-glucoside }\end{array}$ & $\begin{array}{l}\text { 2-Deoxy- } \\
\text { glucose }\end{array}$ \\
\hline JM 1080 & $p t s G^{+} p t s M u m g C^{+}$ & Lactate & $1 \cdot 8$ & $<0.1$ \\
\hline $\mathrm{JM} 1080$ & $p t s G^{+} p t s M$ umg $C^{+}$ & Glucose & $8 \cdot 4$ & ND \\
\hline JM1110 & ptsG $G^{+}$ptsM umgC & Lactate & $9 \cdot 3$ & 0.4 \\
\hline JM1118 & $p t s G$ pts $M$ umgC & Lactate & $<0.01$ & $0 \cdot 3$ \\
\hline JM1138 & ptsG pts $M^{+} u m g C$ & Lactate & 0.03 & 31 \\
\hline
\end{tabular}

assay of methyl $\alpha$-D-glucoside uptake, is still pts $M$ (that is, that it carries a mutation suppressing the glucosamine-negative phenotype of strain JM1080 rather than a reverted $p t s M^{+}$allele) was further demonstrated as follows. Bacteriophage Pl was propagated on the strain and used to transduce strain $\mathrm{JM} 1121$ (eda pts ${ }^{+}$ptsN) to $\mathrm{Eda}^{+}$, selecting for recombinants able to grow on gluconate as sole carbon source. Of 44 such recombinants, $50 \%$ had lost the ability to grow on glucosamine, and must therefore have acquired the pts $M$ allele of the donor strain since $p t s M$ and $e d a$ are cotransducible at about this frequency (Jones-Mortimer \& Kornberg, 1974).

Since our previous results (Kornberg \& Jones-Mortimer, 1975) had indicated that a constitutive PtsG system might transport glucosamine, two types of experiment were carried out to demonstrate the involvement of the PtsG system. A mutant, strain JM1118, was isolated from strain JM1110 as being resistant to glucose whilst growing on glycerol at $41{ }^{\circ} \mathrm{C}$, by the appropriate modification of the method of Jones-Mortimer \& Kornberg (1976). This strain had simultaneously lost the ability to grow on glucosamine and mannose, as is shown in Table 2, which compares the growth of this strain with strains JM1110, JM1080 and JM1138 on glucose, mannose, glucosamine and mannitol, and on lactate in the presence and absence of 2-deoxyglucose. The properties of strain JM1118 show that it is $p t s G$ as well as pts $M$ and indicate that its parent, strain JM1110, uses the PtsG system for the transport of glucosamine, mannose and 2-deoxyglucose.

Strains JM1080, JM1110, JM1118 and JM1138 were assayed for the presence of methyl $\alpha$-D-glucoside and 2-deoxyglucose transport systems during growth on lactate (when the system should not be induced). Strain JM1080 was also assayed for the methyl $\alpha$-D-glucoside transport system after growth on glucose. The results of these experiments (Table 3 ) show that strain JM1118 lacks the methyl $\alpha$-D-glucoside transport system, whereas induced cells of strain JM1080 and uninduced cells of strain JM1110 each have about five times as much transport system activity as uninduced cells of strain JM1080. Of these strains only JM1138 
$\left(p t s M^{+}\right.$) takes up significant quantities of 2-deoxyglucose, even though it fails (being ptsG) to take up methyl $\alpha$-D-glucoside.

The lesion in strain JM1110 was mapped in the following way. Strain HK360 ( $p t s G$ umgC ptsM purB) was transduced with P1 grown on strain DF1071.2B, which forms the PtsG system inducibly, and glucose-positive recombinants were selected. Of 36 of these examined, nine were constitutive for PtsG as judged by the film technique of Kornberg (1972): these grew with mannose or glucosamine as carbon source. The other 27 recombinants were inducible for PtsG and unable to utilize either mannose or glucosamine. (No $\mathrm{PtsM}^{+}$recombinants were observed in this sample.) One of these $p t s G^{+} u m g C^{+}$recombinants which had retained the purB marker was transduced with PI grown on strain JM1110 and $\mathrm{Pur}^{+}$recombinants were selected. Of 160 scored, $16 \%$ were glucosamine-positive. These results show that the lesion in strain JM1110 is linked to $p t s G$ and $p u r B$, and thus that it maps in about the same position as the PtsG-constitutive allele described by Kornberg \& Smith (1972).

These experiments confirm our previous conclusion that glucosamine and mannose can enter $E$. coli by the PtsG system, but are not capable of inducing the system. Though strain $\mathrm{JM} 1110$ ( $p t s G^{+}$pts $M$ umgC) does not take up significant amounts of 2-deoxyglucose when the extracellular concentration is $50 \mu \mathrm{M}$ (Table 3 ), this strain is as effectively inhibited by $10 \mathrm{~mm}$-2-deoxyglucose as is its $p t s G$ pts $M^{+}$derivative (Table 2).

Kornberg \& Smith (1972) isolated a pts $G$ mutant by selecting for resistance to 2-deoxyglucose with fructose as sole carbon source. On the basis of the specificities of the PtsG and PtsM systems, Postma \& Roseman (1976) challenged this procedure and suggested that the selection used should not yield ptsG mutants. The strain in which Kornberg \& Smith (1972) selected their mutant is known to express the PtsG system constitutively (Kornberg \& Reeves, 1972a, b). Fructose, like 2-deoxyglucose, is a substrate for the PtsM transport system (Jones-Mortimer \& Kornberg, 1974; Kornberg \& Jones-Mortimer, 1975) and must therefore be a competitive inhibitor of 2-deoxyglucose translocation by this system. Our results (Table 2) confirm that the selection employed by Kornberg \& Smith (1972) can yield pts $G$ mutants.

That the inability of strain $\mathrm{JM} 1138$ ( $p t s G p t s M^{+} e d a$ ) to grow on glucose is not the result of glucose being unable to enter the cells is clear from the observation that glucose inhibits the growth of the strain on plates with glycerol as carbon source. A ptsM mutant, strain JM1141, derived from strain JM1138 by selection for resistance to glucosamine on medium with glycerol as the carbon source at $41^{\circ} \mathrm{C}$, is also resistant to glucose. We suggest that the reason why strain JM1138 is glucose-negative is that, in strains with a temperature-sensitive fructose1,6-bisphosphate aldolase, this enzyme limits the rate of growth of the strain (Cooper, 1975) and thus leads to high intracellular concentrations of hexose phosphates (in the case of glucose, particularly of glucose 6-phosphate); this consequently leads to a flux through the hexose monophosphate shunt and the dehydratase of the Entner-Doudoroff pathway. Since the strain carries the gnd and eda markers, and thus lacks 6-phosphogluconate dehydrogenase and 2-keto-3-deoxy-6-phosphogluconate aldolase activities, 2-keto-3-deoxy6-phosphogluconate may accumulate. Furthermore, accumulation of 6-phosphogluconate will itself tend to inhibit phosphoglucoisomerase (Muramatsu \& Nosoh, 1971) and so exacerbate the situation.

Comparison of the growth rates of strains JM1077 and JM1080 with glucosamine as sole carbon source (Tables 2 and 4 ) suggests that the gene galP also plays a minor role in glucosamine catabolism. Strain JM 1077 differs from strain JM 1080 only in being galP + (Henderson et al., 1977); it grows on glucosamine poorly but its galP derivative does not grow at all. On solid medium the growth of strain JM 1077 with glucosamine as sole carbon source is not stimulated by the addition of $1 \mathrm{~mm}$-D-fucose, a compound which is known to induce the GalP transport system to about 10 times the basal level (Henderson et al., 1977). This suggests that although the rate of glucosamine uptake may thus be stimulated, the rate of 
Table 4. Mean generation times of $E$. coli strains on different carbon sources

Carbon sources were present at $10 \mathrm{~mm}$. Mean generation times are expressed in $\mathrm{h}$.

$\begin{array}{lcccc}\text { Strain } & \text { Genotype } & \text { Mannitol } & \begin{array}{c}\text { Glucos- } \\ \text { amine }\end{array} & \begin{array}{c}\text { Carbon -Acetyl- } \\ \text { glucosamine }\end{array} \\ \text { JM1077 } & \text { ptsM pts } N^{+} & 2 \cdot 3 & 9 & 1 \cdot 9 \\ \text { JM1120 } & \text { ptsM ptsN } & 2 \cdot 0 & 13 & >48 \\ \text { JM1121 } & \text { ptsM } M^{+} \text {pts } N & 1 \cdot 8 & 2 \cdot 5 & 2 \cdot 4\end{array}$

phosphorylation of glucosamine taken up by this route, presumably by a soluble, ATPdependent kinase as described by White (1968), may be limiting the rate of growth under these conditions. Roehl \& Vinopal (1976) have shown that mannose may also enter $E$. coli by the GalP transport system.

\section{Phosphotransferase systems for $N$-acetylglucosamine}

Preliminary experiments indicated that though $N$-acetylglucosamine-negative mutants could readily be isolated, by the appropriate modification of the method of Jones-Mortimer $\&$ Kornberg (1976), from $f d a^{\text {ts }}$ strains that were also $p t s M$, they could not be isolated when such strains were $p t s M^{+}$. The system appeared analogous to the one we have previously described (Jones-Mortimer \& Kornberg, 1974) where $f p k p f k$ double mutants are unable to mutate spontaneously to resistance to $20 \mathrm{~mm}$ fructose on glycerol since two independent mutational events would be required. This suggested that the PtsM system might be identical to one of the phosphotransferase activities described by White (1970) as capable of transporting $N$-acetylglucosamine.

In order to test this hypothesis, we isolated from the $f d a^{\text {ts }} p t s M$ strain JM1077 a mutant (strain JM1120) which was resistant to $N$-acetylglucosamine at $41{ }^{\circ} \mathrm{C}$ and was unable to grow at $30^{\circ} \mathrm{C}$ with $\mathrm{N}$-acetylglucosamine as sole carbon source. Table 4 compares the growth of this strain with that of its parent. Although both grow well on mannitol and both grow poorly on glucosamine, only strain JM1077 grows readily on $\mathrm{N}$-acetylglucosamine.

When bacteriophage P1 grown on strain DF1071.2B ( $p t s M^{+} e d a$ ) was used to transduce strain $\mathrm{JM} 1120$ to pts $^{+}, 30 \%$ of the recombinants selected on glucosamine were gluconatenegative and had thus inherited the eda allele from the donor strain. All recombinants from this cross were $\mathrm{N}$-acetylglucosamine-positive; one which was also gluconate-negative (strain JM1121, Tables 1 and 4) was used in an experiment described earlier in this paper. This experiment, the transduction of pts $M$ from strain JM1110 into strain JM1121 selecting for $\mathrm{Eda}^{+}$recombinants yielded, as previously stated, glucosamine-negative recombinants. These recombinants proved without exception to be unable to utilize $N$-acetylglucosamine as sole carbon source. These results show that the gene pts $M$ codes for a phosphotransferase capable of transporting $\mathrm{N}$-acetylglucosamine and that the gene for the second $\mathrm{N}$-acetylglucosamine transport system is not closely linked to $p t s M$ and $e d a$.

We therefore set out to test White's (1970) suggestion that the gene for the other phosphotransferase $(p t s N)$ was linked to the genes $n a g A, B$ which specify the enzymes for the conversion of $N$-acetylglucosamine 6-phosphate to fructose 6-phosphate. Transduction of strain JM1120 with bacteriophage P1 grown on the glt A strain K1.1.4 and selection for galactose-positive recombinants yielded no $N$-acetylglucosamine-positive recombinants among the 385 colonies scored, though $29 \%$ of these were glt $A$. Therefore either the galK and $p t s N$ loci are not cotransducible, or strain $\mathrm{K} 1.1 .4$ does not carry the $p t s N^{+}$allele. Preliminary experiments with a recombinant from this cross, JM1123 (glt A ptsN), indicated that these genes could be cotransduced, but that neither marker was stable enough 
Table 5. Uptake of $\mathrm{N}$-acetyl $\left[{ }^{14} \mathrm{C}\right]$ glucosamine by $\mathrm{E}$. coli strains grown with lactate as the carbon source in the presence or absence of $N$-acetylglucosamine

\begin{tabular}{|c|c|c|c|}
\hline Strain & NAG* & Genotype & $\begin{array}{c}\text { Uptake of }\left[{ }^{14} \mathrm{C}\right] \mathrm{NAG} \\
{\left[\mathrm{nmol}\left(\mathrm{mg} \mathrm{dry} \mathrm{wt}^{1}\right.\right.} \\
\left.\mathrm{min}^{-1}\right]\end{array}$ \\
\hline JM 1077 & - & $p t s M p t s N^{+}$ & $10 \cdot 2$ \\
\hline JM1077 & + & $p t s M p t s N^{+}$ & $12 \cdot 8$ \\
\hline JM1121 & - & $p t s M^{p t s N}$ & $8 \cdot 8$ \\
\hline JM1121 & + & $p t s M^{+} p t s N$ & 8.9 \\
\hline JM1120 & - & $p t s M p t s N$ & $1 \cdot 8$ \\
\hline JM1111 & - & $p t s M p t s N^{t}$ & $14 \cdot 2$ \\
\hline JM1161 & - & $p t s M$ pts $N^{+}$ & $13 \cdot 2$ \\
\hline JM1158 & - & $p t s M p t s N$ & $<0.1$ \\
\hline JM1165 & - & $p t s M p t s N$ & $<0.1$ \\
\hline JM1166 & - & $p t s M$ pts $N$ & $<0 \cdot 1$ \\
\hline
\end{tabular}

to obtain reliable cotransduction frequencies. We therefore attempted to measure the cotransduction between $p t s N$ and supE. Phage Pl grown on strain 5K-C600 (supE) was used to transduce strain JM1158 (pts $N$ pts $M c y s I$ ), which is $N$-acetylglucosamine-negative and a cystine auxotroph because of an amber mutation in cysI. Of $350 \mathrm{~N}$-acetylglucosaminepositive recombinants scored, 102 were glucosamine-positive (i.e. PtsM ${ }^{+}$) and cystinenegative. Of the remainder, 53 were cystine-negative and 195 grew in the absence of cystine at $30{ }^{\circ} \mathrm{C}$ (but not at $40^{\circ} \mathrm{C}$ : presumably the suppressed cys $I$ gene product is thermolabile). This result indicates either that the cotransduction frequency of pts $N$ with $\sup E$ is $79^{\circ}{ }_{0}$, or that the $p t s N$ allele in strain JM1158 is suppressible by supE. To distinguish between these hypotheses we took a recombinant from this cross, strain JM1161, which was $\mathrm{Nag}^{+} \mathrm{Cys}$ and from it isolated two independent $N$-acetylglucosamine-negative mutants, strains JM1165 and JM1166. When P1 grown on strain JM1077 (nag ${ }^{+} \sup E^{+}$ptsM) was used to transduce strains JMI165 and JMI166 to $\mathrm{Nag}^{+}$the majority of the recombinants tested, $60 \%$ and $75 \%$ respectively, had lost the ability to grow at $30{ }^{\circ} \mathrm{C}$ in the absence of cystine. We therefore conclude that $p t s N$ and supE are cotransducible. Therefore the gene for the second $N$-acetylglucosamine phosphotransferase maps near $\operatorname{nag} A, B$, as predicted by White (1970).

This result was substantiated by the isolation at $40^{\circ} \mathrm{C}$ from strain $\mathrm{JM} 1110$ ( fda pts $\mathrm{M}$ $u m g C$ ) of an $N$-acetylglucosamine-resistant derivative, strain JM1156, which had simultaneously become sensitive to glucosamine at $30^{\circ} \mathrm{C}$. Since this strain, unlike other $N$-acetylglucosamine-negative strains, does not mutate to grow on $\mathrm{N}$-acetylglucosamine we consider that it is likely to have a deletion of the genes $p t s N$ and $\operatorname{nag} B$ (the latter specifying glucosamine-6-phosphate deaminase) and possibly also of $n a g A$ (the gene for $N$-acetylglucosamine-6-phosphate deacetylase). The lesion is cotransducible with supE: of $16 \mathrm{~N}$-acetylglucosamine-positive transductants obtained with strain 5K-C600 as the donor, 14 were $\sup E$, as judged by the ability of the T4 amber mutant $\mathrm{N} 82$ to form plaques on them.

The results presented in Table 5 confirm that the inability of pts $M$ pts $N$ double mutants to grow with $\mathrm{N}$-acetylglucosamine as sole carbon source (Table 4) is due to a defect in the transport of the sugar, and demonstrate that the amount of sugar transported by either system is sufficient to account for the observed rate of growth. Neither transport system was significantly induced by growth in the presence of $\mathrm{N}$-acetylglucosamine.

These results illuminate the finding of White \& Kent (1970) and White (1970) that $\mathrm{N}$ acetylglucosamine transport-deficient mutants could be isolated by two-stage selection for resistance to $\mathrm{N}$-iodoacetylglucosamine, if the first selection was carried out with glucose as the carbon source. Under these conditions the toxic analogue would presumably not be taken up by the PtsM transport system (since glucose also enters by this system) so that 
mutants which have lost the $N$-acetylglucosamine transport system should readily be obtained. These mutants, however, will remain sensitive to the toxic analogue during growth on carbon sources which are not substrates for the PtsM system, but resistant mutants selected from such a strain will, by becoming $p t s M$, lose the ability to transport $N$-acetylglucosamine. This hypothesis was tested by isolating from strain $5 \mathrm{~K}-\mathrm{C} 600 \mathrm{a}$ mutant, strain JM1179, resistant to 2-deoxy-2-iodoacetamidoglucose during growth on glucose, by the method of White \& Kent (1970). Bacteriophage P1 grown on this strain was used to transduce strain JM1156, selecting for recombinants on glucosamine as sole carbon source. Of eight transductants analysed all were $\mathrm{N}$-acetylglucosamine-negative at $30^{\circ} \mathrm{C}$ and resistant to 2-deoxy-2-iodoacetamidoglucose on lactate medium in the presence of glucose. In a control experiment with strain JM1138 as recipient, of $120 \mathrm{Eda}^{+}$recombinants tested, none was resistant to the analogue. Therefore, the conditional analogue-resistant mutants of the type described by White \& Kent (1970) have lost the $N$-acetylglucosamine transport system specified by the gene $p t s N$.

Mutants deficient in glucosamine-6-phosphate synthetase $(\mathrm{g} / \mathrm{m} S)$ require glucosamine or $N$-acetylglucosamine for growth (Wu \& Wu, 1971; Imada et al., 1977). In the presence of glucose only $\mathrm{N}$-acetylglucosamine satisfies their growth requirement. Imada et al. (1977) suggested that this is because glucose inhibits the phosphotransferase for glucosamine but not that for $\mathrm{N}$-acetylglucosamine. Our results indicate that, since glucosamine must enter cells by either the PtsM or PtsG system, glucose should be a competitive inhibitor of both uptake systems for glucosamine, though it need not be an inhibitor of the PtsN system for $\mathrm{N}$-acetylglucosamine for which it is not a substrate.

This work was supported by The Science Research Council under grant B/RG/9756.2.

\section{REFERENCES}

Ashworth, J. M. \& Kornberg, H. L. (1966). The anaplerotic fixation of carbon dioxide by Escherichia coli. Proceedings of the Royal Society $\mathbf{B 1 6 5}$ 179-188.

BaChMan, B. J., Low, K. B. \& TAYlor, A. L. (1976). Recalibrated linkage map of Escherichia coli K12. Bacteriological Reviews 40, 116-167.

CoOper, R. A. (1975). The methylglyoxal by-pass of the Embden-Meyerhof pathway. Biochemical Society Transactions 3, 837-840.

Curtis, S. J. \& Epstein, W. (1975). Phosphorylation of D-glucose in Escherichia coli mutants defective in glucose phosphotransferase, mannose phosphotransferase and glucokinase. Journal of Bacteriology 122, 1189-1199.

Faik, P., KornberG, H. L. \& McEvoy-Bowe, E. (1971). Isolation and properties of Escherichia coli mutants defective in 2-keto-3-deoxy-6-phosphogluconate aldolase activity. FEBS Letters 19, 225228.

Henderson, P. J. F., Giddens, R. A. \& JonesMortimer, M. C. (1977). Transport of galactose, glucose and their molecular analogues by Escherichia coli K12. Biochemical Journal 162, 309320.

Imada, A., Nozaki, Y., Kawashima, F. \& Yonida, M. (1977). Regulation of glucosamine utilization in Staphylococcus aureus and Escherichia coli. Journal of General Microbiology 100, 329-337.

Jones-Mortimer, M. C. (1973). Mapping of structural genes for the enzymes of cysteine biosyn- thesis in Escherichia coli $\mathrm{K} 12$ and Salmonella typhimurium LT2. Heredity 31, 213-221.

Jones-Mortimer, M. C. \& KorNBerG, H. L. (1974). Genetical analysis of fructose utilisation by Escherichia coli. Proceedings of the Royal Society B187 121-131.

Jones-Mortimer, M. C. \& KornberG, H. L. (1976). Order of genes adjacent to pts $X$ on the $E$. coli genome. Proceedings of the Royal Society B193, 313-315.

KORNBERG, H. L. (1972). Nature and regulation of hexose uptake by Escherichia coli. In The Molecular Basis of Biological Transport, Miami Winter Symposia, vol. 3, pp. 157-180. Edited by J. F. Woessner, Jr \& F. Huijing. New York \& London: Academic Press.

KoRNBERG, H. L. (1976). Genetics in the study of carbohydrate transport by bacteria. Journal of General Microbiology 96, 1-16.

Kornberg, H. L. \& Jones-Mortimer, M. C. (1975). $P t s X$ : a gene involved in the uptake of glucose and fructose by Escherichia coli. FEBS Letters 51, $1-4$.

Kornberg, H. L. \& Reeves, R. E. (1972a). Correlation between hexose transport and phosphotransferase activity in Escherichia coli. Biochemical Journal 126, 1241-1243.

KornberG, H. L. \& ReEves, R. E. (1972b). Inducible phosphoenolpyruvate-dependent hexose phosphotransferase activites in Escherichia coli. Biochemical Journal 128, 1339-1344. 
KornberG, H. L. \& Smith, J. (1972). Genetic control of glucose uptake by Escherichia coli. FEBS Letters 20, 270-272.

Kundic, W., Ghosh, S. \& Roseman, S. (1964). Phosphate bound to histidine in a protein as an intermediate in a novel phosphotransferase system. Proceedings of the National Academy of Sciences of the United States of America 52, 1067-1074.

LenNox, E. S. (1955). Transduction of linked genetic characters of the host by bacteriophage $\mathrm{Pl}$. Virology 1, 190-206.

Muramatsu, N. \& Nosoh, Y. (1971). Purification and characterization of glucose-6-phosphate isomerase from Bacillus stearothermophilus. Archives of Biochemistry and Biophysics 144, 245-252.

Postma, P. W. \& Roseman, S. (1976). The bacterial phosphoenolpyruvate:sugar phosphotransferase system. Biochimica et biophysica acta 457, 213257.

RoEHL, R. A. \& Vinopal, R. T. (1976). Lack of glucose phosphotransferase function in phosphofructokinase mutants of Escherichia coli. Journal of Bacteriology 126, 852-860.

White, R. J. (1968). Control of amino sugar metabolism in Escherichia coli and isolation of mutants unable to degrade amino sugars. Biochemical Journal 106, 847-858.

White, R. J. (1970). The role of the phosphoenolpyruvate phosphotransferase system in the transport of $N$-acetyl-D-glucosamine by Escherichia coli. Biochemical Journal 118, 89-92.

White, R. J. \& Kent, P. W. (1970). An examination of the inhibitory effects of $N$-iodoacetylglucosamine on Escherichia coli and isolation of resistant mutants. Biochemical Journal 118, 81-87.

WU, H. C. \& WU, T. C. (1971). Isolation and characterisation of a glucosamine-requiring mutant of Fscherichia coli $\mathrm{K} 12$ defective in glucosamine-6phosphate synthetase. Journal of Bacteriology 105 , 455-466. 